
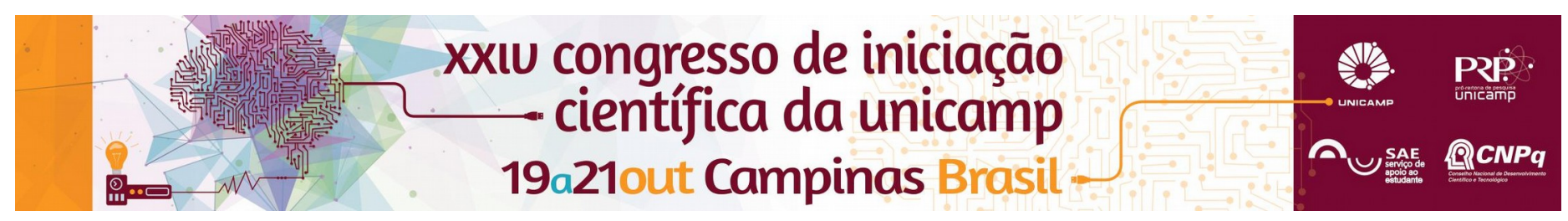

\title{
First Order Logic and Gödel's Theorems
}

\section{Abner M. Brito*, Fabio M. Bertato}

\begin{abstract}
A study of First Order Mathematical Logic, covering the basics of Statement and Predicate Calculus: the ground on which First Order Theories are built. Completeness and consistency of such theories. Conclusion that number theory cannot be both consistent and complete.
\end{abstract}

\section{Key words:}

Mathematical Logic, First Order Logic, Gödel's Theorems

\section{Introduction}

First Order Logic (FOL) is a systematic approach to the foundations of much of mathematics. It is presented as the fundamental building block on which mathematical derivation can be built, leaving no room for ambiguity or uncertainty. Number theory and set theory can be axiomatized as first order theories.

This research was aimed at giving introductory comprehension of the grounds of proof theory, as well as an understanding of the limitedness of mathematics.

\section{Results and Discussion}

FOL is formulated in such a way that every theorem can be derived from axioms alone, using modus ponens as its sole rule of inference. Although finding a proof for a theorem may be challenging, verifying the correctness of a formal proof is an automatic process that can be performed by a computer. FOL is composed of the Statement Calculus (SC) and the Predicate Calculus (PC). Every theorem of the statement calculus is a theorem of the predicate calculus.

Making assertions about FOL and its theorems however is a different matter. One cannot speak about a formal language or a formal theory from within that formal language. A metalanguage, such as English or Portuguese is necessary. In a sense metatheorems theorems in metalanguage, about the formal theory - are central in studying formal logic. And it is precisely these metatheorems that constitute the core of the research developed.

The main metatheorems of SC are the deduction theorem and the completeness theorem for the statement calculus. The deduction theorem is a formalizable justification (for each theorem) of the mathematical custom of supposing $A$, deriving $B$ and concluding that $A$ implies $B$, whereas the completeness theorem (for SC) states that every tautology is a theorem.

Moving on to PC, we develop rules for introducing and removing the universal and existential quantifiers ( $\forall$ and $\exists$, respectively) and for replacing a subformula of a given formula by an equivalent subformula. $\mathrm{PC}$ is then provided with equality axioms.

Once PC (with equality) is established we may specify some operation and predicate symbols and add new statements to our set of (proper) axioms to define a First Order Theory (FOT). After defining and proving basic theorems of Number Theory $(\mathrm{N})$, we develop the concepts of consistency and completeness of a FOT, including the completeness theorem (for consistent FOTs) and the Löwenheim-Skolem theorem. Finally, from the concept of decidability we sketch a proof of Gödel's incompleteness theorem, concluding that $\mathrm{N}$ cannot be both consistent and complete.

\section{Conclusions}

First Order Logic is a solid foundation on which we can build much of mathematics. A formal proof in FOL is accurate, and leaves us with no room for doubt - as long as we have previously accepted the basic axioms and rule of inference. However, it also reveals that not every mathematical statement is necessarily true or false. In number theory, consistency implies incompleteness. And if a statement can be neither true nor false, if the law of excluded middle - which permeates the whole of Western classical thought - is not necessary, our notion of proof may be weakened, opening new possibilities for mathematical reasoning.

\section{Acknowledgement}

This research project was financially supported by PIBIC - SAE/UNICAMP.

[1] Margaris A. First order mathematical logic. Dover; 1990.

[2] Carnielli W, Epstein RL. Computabilidade, funções computáveis, lógica e os fundamentos da matemática. Editora UNESP; 2006.

[3] Shoenfield JR. Mathematical logic. Association for Symbolic Logic; 1967.

[4] Mendelson E. Introduction to mathematical logic. Chapman and Hall; 2009.

[5] Lourenço M, editor. O Teorema de Gödel e a hipótese do contínuo. Fundação Calouste Gulbenkian; 1979. 